https://doi.org/10.23913/ciba.v8i16.92

Artículos Científicos

\title{
Relaciones talla-peso y factor de condición de la tilapia Orecochromis niloticus en cinco cuerpos de agua del estado de Jalisco, México
}

Size-weight relationship and condition factor of tilapia Orecochromis niloticus in five water bodies of Jalisco State, Mexico

Razões altura / peso e fator de condição de Orecochromis niloticus tilapia em cinco massas de água no estado de Jalisco, México

Felipe Santoyo Telles

Universidad de Guadalajara, Centro Universitario del Sur, México

felipes@cusur.udg.mx https://orcid.org/0000-0003-3854-9405

José Mariscal Romero

Universidad de Guadalajara, Centro Universitario del Sur, México mariscal@costeramelaque.udg.mx http://orcid.org/0000-0003-0978-0480

Carlos Gómez Galindo

Universidad de Guadalajara, Centro Universitario del Sur, México carlosg@cusur.udg.mx https://orcid.org/0000-0002-6257-7337

Humberto Gutiérrez Pulido

Universidad de Guadalajara, Departamento de Matemáticas, México humpulido@yahoo.com https://orcid.org/0000-0003-1943-6712 
Revista Iberoamericana de las Ciencias Biológicas

y Agropecuarias

\section{Resumen}

En este trabajo se analizaron las relaciones talla-peso y factor de condición de 3606 individuos de Oreochromis niloticus colectados desde enero hasta diciembre de 2010 en cinco cuerpos de agua: dos lagos endorreicos, conocidos como lagunas de Zapotlán y de Cajititlán, y tres embalses artificiales de capacidades similares, denominados presas Calderón, El Salto y Basilio Vadillo. También se exploraron las relaciones entre las variables morfométricas mencionadas con variables de calidad del hábitat como temperatura, oxígeno, pH, potencial óxido reducción (ORP) y sólidos disueltos totales (TDS). El análisis del estado nutricional de los individuos medido a través del índice factor de condición demuestra que los peces que habitan los lagos de Zapotlán y Cajititlán son significativamente más pequeños y con una condición más pobre que los que se hallan en las presas Calderón y El Salto, con la excepción de B. Vadillo, la cual presenta valores semejantes a los lagos. Los valores bajos del factor de condición en los lagos se asocian con niveles bajos de temperatura y concentración de oxígeno disuelto, valores altos de $\mathrm{pH}$ y sólidos disueltos, así como un valor bajo en el potencial de óxido reducción, atribuidos a la descarga de aguas residuales de las áreas urbanas adyacentes, con una carga orgánica que afecta la calidad del hábitat acuático e impacta la condición de los individuos. En contraste, las tilapias de mejor talla-peso y condición se capturaron en las presas de El Salto y Calderón, donde los niveles de oxígeno son más elevados, $\mathrm{pH}$ cercano al neutro, menor cantidad de sólidos disueltos y potencial óxido reductor positivo. Se asume que talla, peso y factor de condición (K) de los individuos de Orechromis niloticus varían en función de la calidad del hábitat.

Palabras clave: aspectos bioecológicos, hidrología, oreochromis niloticus.

\section{Abstract}

Variations of size -weight relationships and condition factor of 3,606 individuals of Oreochromis niloticus, collected from January to December 2010, in five water bodies, of Jalisco Mexico, were analyzed. Water bodies include two endorrheic lakes, known as "Laguna: de Zapotlán" and "Laguna de Cajititlán", and three artificial reservoirs of similar capacities, known as Calderón, El Salto and Basilio Vadillo dams. Relationships between the mentioned morphometric variables and habitat quality variables, such as temperature, oxygen, $\mathrm{pH}$, oxide reduction potential (ORP) and total dissolved solids (TDS) were also 
Revista Iberoamericana de las Ciencias Biológicas y Agropecuarias

explored. Analysis of the nutritional status of the individuals measured through the condition factor index, indicates that fish that inhabit Zapotlán and Cajititlán lakes are significantly smaller and with a poorer condition than those that live in the Calderón and El Salto dams, with the exception of Basilio Vadillo that presents values similar to lakes. Low values of the condition factor in the lakes are associated with low values of temperature and dissolved oxygen, high values for $\mathrm{pH}$ and dissolved solids, and low oxide reduction potential, attributed to the discharge of wastewater from the adjacent urban areas, whose organic load affects the quality of the aquatic habitat and impacts the condition of the individuals. In contrast, greater tilapia sizes, of better weight and condition, were captured in El Salto and Calderón dams, where oxygen levels are higher, $\mathrm{pH}$ close to neutral, fewer dissolved solids and a positive oxide reductive potential. It is assumed that size, weight and condition factor $(\mathrm{K})$ of the individuals of Orechromis niloticus, vary depending on the quality of the habitat.

Keywords: bio-ecological aspects, multivariate analysis, hydrology, oreochromis niloticus.

\section{Resumo}

Neste trabalho, foram analisadas as relações altura-peso e fator de condição de 3606 indivíduos de Oreochromis niloticus coletados de janeiro a dezembro de 2010 em cinco corpos d'água: dois lagos endorréicos, conhecidos como lagoas de Zapotlan e Cajititlán, e três reservatórios artificiais de capacidades similares, denominadas barragens de Calderón, El Salto e Basilio Vadillo. Também foram exploradas as relações entre as variáveis morfométricas mencionadas e as variáveis de qualidade do habitat, como temperatura, oxigênio, pH, redução potencial de óxido (ORP) e sólidos totais dissolvidos (TDS). A análise do estado nutricional dos indivíduos medidos pelo índice de fator de condição mostra que os peixes que habitam os lagos de Zapotlán e Cajititlán são significativamente menores e com uma condição mais pobre do que os encontrados nas barragens de Calderón e El Salto, com exceção de B. Vadillo, que apresenta valores semelhantes aos dos lagos. Os baixos valores do fator de condição nos lagos estão associados a baixos níveis de temperatura e concentração de oxigênio dissolvido, altos valores de $\mathrm{pH}$ e sólidos dissolvidos, além de um baixo valor no potencial de redução de óxido, atribuído à descarga de águas residuais de áreas urbanas adjacentes, com uma carga orgânica que afeta a qualidade do habitat aquático e afeta a condição dos indivíduos. Por outro lado, foram capturadas tilápias com melhor altura e 
condição nas barragens de El Salto e Calderón, onde os níveis de oxigênio são mais altos, pH próximo ao neutro, menor quantidade de sólidos dissolvidos e potencial redução de óxido positivo. Supõe-se que tamanho, peso e fator de condição $(\mathrm{K})$ de indivíduos de Orechromis niloticus variam de acordo com a qualidade do habitat.

Palavras-chave: aspectos bioecológicos, hidrologia, oreochromis niloticus.

Fecha recepción: Marzo 2019

Fecha aceptación: Julio 2019

\section{Introducción}

La exploración de las relaciones biométricas, particularmente las que se refieren a la longitud-peso y el factor de condición $(\mathrm{K})$, son descriptores de gran interés en la biología de las poblaciones (Valencia-Santana y Valencia-Santana, 2015), sobre todo de organismos ectotermos como los peces, ya que estos por su condición (dependientes de factores ambientales) son muy susceptibles a las variaciones y cambios, lo cual puede aportar información fundamental sobre su crecimiento y su estado nutricional y reproductivo. Asimismo, son parámetros ampliamente utilizados para comparar la condición de poblaciones que habitan en sistemas acuáticos con distintos grados de perturbación (Cifuentes et al., 2012). Por ello, conocer la información comparativa básica de estas poblaciones acuáticas que habitan en ecosistemas prístinos o perturbados es muy importante para entender la dinámica de los cambios o los efectos en otras especies sometidas a impactos antropogénicos.

Por otro lado, y en cuanto a las cuencas, se puede decir que representan divisiones naturales del paisaje cuyo funcionamiento ecohidrológico se sustenta en un equilibrio frágil y dinámico, por lo que cualquier alteración en algunos de sus componentes modificará la dinámica de los ciclos hidrológicos y biogeoquímicos, lo que se verá reflejado en la condición ecológica de los peces y en la calidad ambiental de los cuerpos de agua. De acuerdo con un diagnóstico reciente (Cotler, 2010), en $66 \%$ de las cuencas del país la alteración de su dinámica ha producido un deterioro de alto a extremo en su funcionamiento, entre las que se incluyen las de Zapotlán, Cajititlán, así como las de las presas Basilio Vadillo, El Salto y Calderón, donde habitan las poblaciones de Oreochromis analizadas. 
Ahora bien, la tilapia del Nilo (Oreochromis niloticus) es una especie exótica con altos rendimientos para la pesquería comercial y de consumo local, de ahí que sea una alternativa económica y nutricional para las comunidades rurales de México. Además, por su alta tolerancia y sus estrategias adaptativas ha sido introducida y proliferado en más de 100 países.

Por estas razones, el objetivo de este trabajo es explorar las relaciones biométricas talla-peso para determinar de qué manera el factor de condición fisiológica o de bienestar de las tilapias se ve afectado por la variación en las condiciones ambientales de esos cinco cuerpos de agua.

\section{Área de estudio}

\section{Cuenca Lerma-Chapala-Santiago}

Los cinco cuerpos de agua del área de estudio se ubican en la región hidrológica VIII Lerma-Santiago-Pacífico, en la subregión hidrológica Armería-Coahuayana (figura 1). Esta área está constituida por dos lagos naturales (Zapotlán y Cajititlán, cuyas características son endorreicas) y tres embalses artificiales o presas (Basilio Badillo, Calderón y El Salto, con características exorreicas). Se consideró relevante el estudio comparativo, ya que son cuerpos de agua satélites de las regiones Lerma-Chapala-Santiago y Ayuquila-Armeria, señaladas como prioritarias por requerir medidas urgentes para recuperar su funcionamiento ecohidrológico (Cotler, 2010). 
Figura 1. Área de estudio (localización de los cinco cuerpos de agua)

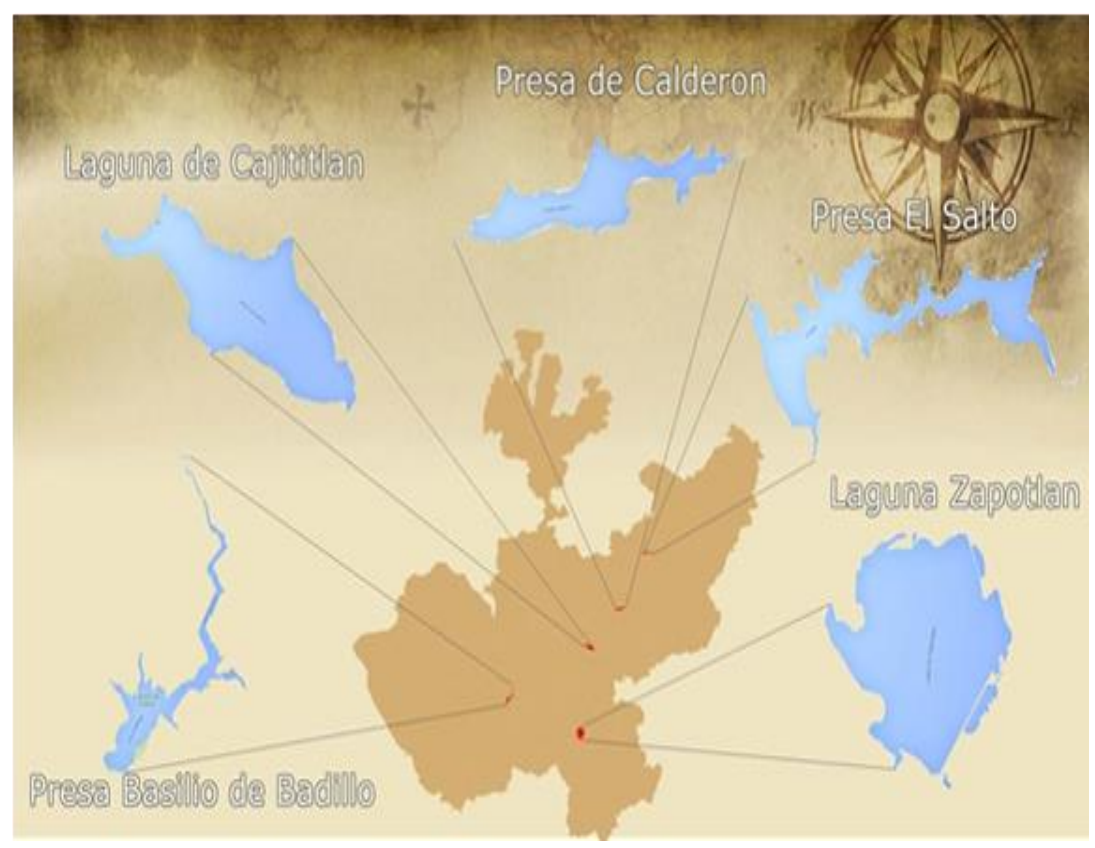

Fuente: Elaboración propia

El lago Zapotlán (conocido como laguna de Zapotlán) se localiza al sur de Jalisco, en la parte occidental de México. Este pertenece a la subcuenca del mismo nombre y tiene una extensión aproximada de $499 \mathrm{~km}^{2}$, con una profundidad promedio de $4.75 \mathrm{~m}$ y una evaporación media anual de $17.7 \mathrm{~mm}^{3}$ (Comisión Estatal del Agua del Estado de Jalisco [CEA], 2015).

El lago Cajititlán (también conocido como Laguna) se encuentra dentro de la zona metropolitana de Guadalajara; tiene $9 \mathrm{~km}$ de longitud, $2 \mathrm{~km}$ de ancho y una profundidad máxima de $4 \mathrm{~m}$. Su capacidad de almacenamiento estimada es de $54400 \mathrm{~mm}^{3}$ en un área de 1700 hectáreas.

La presa Basilio Badillo se ubica en el municipio de Ejutla, Jalisco, a unos $25 \mathrm{~km}$ al noreste de la población de El Grullo, y cuenta con un espejo de agua de 500 ha, con dos vértices de longitud (4.67 y 5.55) y anchuras de $2.06 \mathrm{~km}$ y $0.89 \mathrm{~km}$, con capacidad de 85000 $\mathrm{mm}^{3}$.

La presa Elías González Chávez (conocida como presa de Calderón) se halla en el municipio de Zapotlanejo, en Jalisco. Posee un espejo de agua de 382 ha, con longitud de $6.15 \mathrm{~km}, 1.56 \mathrm{~km}$ de ancho y $80000 \mathrm{~mm}^{3}$ de capacidad. 
La presa El Salto se localiza en el municipio de Valle de Guadalupe, en Los Altos de Jalisco, a $12 \mathrm{~km}$ de distancia de la cabecera municipal; con un espejo de agua de 959 ha, longitud de $8.58 \mathrm{~km}$ y anchos variables (entre $3.73 \mathrm{~km}, 2.20 \mathrm{~km}$ y $1.76 \mathrm{~km}$ ) y una capacidad de $85000 \mathrm{~mm}^{3}$.

Del análisis de las microcuencas donde se ubican los cinco sitios estudiados se determinó que las tres presas son microcuencas exorreicas, mientras que las de los lagos son del tipo endorreico. Además, aunque las áreas de captación y longitudes de los cauces principales relacionados con las tres presas son diferentes, sus volúmenes de almacenamiento son similares (presa Calderón 80 000, mientras que Basilio Vadillo y El Salto $85000 \mathrm{~mm}^{3}$ ). En cambio, los lagos naturales cuentan con diferentes capacidades de almacenamiento (Cajititlán $54400 \mathrm{~mm}^{3}$ y Zapotlán $27000 \mathrm{~mm}^{3}$ ).

Los tiempos de concentración relacionados a la longitud y pendiente media del cauce son relativamente cortos, no mayores a 4 horas en los lagos naturales, mientras que para los embalses oscilan entre 8 y 19.4 horas. Asimismo, existen ciertas similitudes en las superficies totales de captación entre las cuencas de laguna de Zapotlán y presa Calderón, aunque la capacidad de almacenamiento de dicha "laguna" es la menor de todo el conjunto de los cinco cuerpos de agua. Si se considera la relación volumen de almacenamiento $\left(\mathrm{mm}^{3}\right) /$ superficie (ha), las presas estudiadas son más profundas, pues tienen valores que oscilan entre 88-209, mientras que los lagos son más someros, con un rango de valores de tan solo 27-32.

Las poblaciones cercanas a los lagos estudiados tienen una influencia profunda en el deterioro de su ambiente acuático, ya que ambos lagos (Zapotlán y Cajititlán) reciben descargas directas de aguas residuales. Esto ha afectado seriamente las variables ambientales del agua, la relación DBO/DQO, lo que ha favorecido la proliferación de malezas acuáticas (como el lirio Eichornia crassipes) que bloquean la fotosíntesis acuática, aumentan la evapotranspiración y provocan la pérdida de la calidad del hábitat para organismos acuáticos. Esta eutrofización/distrofia afecta la pesca, la navegación y el turismo, lo que genera amplias pérdidas económicas. En cuanto a la presencia de maleza acuática superficial, los lagos tuvieron una cobertura de lirio similar, con promedios anuales de entre $14 \%$ y $17 \%$. 


\section{Materiales y métodos}

Los datos biométricos (talla, longitud total y peso) fueron medidos en 3606 ejemplares frescos de tilapia O. niloticus (Linnaeus 1758) procedentes de las capturas comerciales en los cinco cuerpos de agua mencionados. La captura se realizó con redes agalleras de 3.5 pulgadas, entre los meses de enero y diciembre del año 2010. Para la caracterización físico-química del agua de los cinco embalses, se midieron seis variables mediante una sonda YSI-556, en 4-8 estaciones georreferenciadas y a tres profundidades fondo $(-20 \mathrm{~cm})$, media agua y superficie $(-20 \mathrm{~cm})$. Las variables fueron temperatura $\left({ }^{\circ} \mathrm{C}\right)$, potencial hidrógeno $(\mathrm{pH})$, oxígeno disuelto $[\mathrm{mg} / \mathrm{l}]$, potencial de óxido-reducción (ORP), sólidos totales disueltos (DST) y salinidad.

\section{Análisis de datos}

\section{Factor de condición de Fulton (K)}

El factor de condición $(\mathrm{K})$ es utilizado en peces para medir la relación volumétrica en función del peso, ya que permite no solo comparar peces de las mismas longitudes, sino también determinar el grado de bienestar o robustez (Martínez, 1987). Esto se estima mediante la siguiente expresión:

$$
\mathrm{k}=100 * \mathrm{WL}^{3}
$$

donde $W$ es el peso corporal húmedo en gramos y $L$ la longitud en centímetros. Se analizó la variación mensual entre los cuerpos de agua del factor K mediante un test Anova.

\section{Relación entre longitud y el peso}

Para definir la relación entre el peso y la longitud, los datos fueron ajustados mediante un modelo de potencia calculado por el método de mínimos cuadrados.

$$
\mathrm{W}=\mathrm{A}^{*} \mathrm{Lb}
$$

donde $W$ es el peso en gramos y $L$ la longitud en centímetros (Da Costa y Gerson, 2003). El coeficiente $b$ proporciona información acerca del tipo de crecimiento que exhibe la 
especie; si $\mathrm{b}=3$, el crecimiento es isométrico, $\mathrm{y}$ cuando $\mathrm{b} \neq 3$, es alométrico (Bagenal y Tesch, 1978; Ricker, 1975). Cuando b > 3, los individuos de mayor talla incrementan su peso en mayor proporción que su longitud (alometría positiva); cuando $b<3$, se incrementa preferencialmente la longitud más que su peso (alometría negativa); además, se consideran especies de crecimiento isométrico las que fluctúan dentro de los valores $b=2.5$ y $b=3.5$ (Carlander, 1969; Froese, 2006).

\section{Resultados}

La composición por tallas en la captura de tilapia presenta un sesgo debido a la selectividad de las redes que se utilizaron en las capturas de las muestras. En concreto, $92.48 \%$ de la captura se basa en las tallas de $18 \mathrm{~cm}$ a $24 \mathrm{~cm}$ de longitud total, es decir, individuos adultos (tabla 1). Los promedios de peso y talla medidos en los cinco cuerpos de agua $(p<0.005)$ exhiben diferencias estadísticamente significativas $(p<0.5)$, en los datos agrupados por temporadas (trimestres). 
Tabla 1. Valores descriptivos de los peces

\begin{tabular}{|c|c|c|c|c|}
\hline Lugar & Sexo & & Media & Desv. típ. \\
\hline \multirow{4}{*}{ Zapotlán } & \multirow{2}{*}{ Macho } & L total & 22.71 & 1.17 \\
\hline & & Peso & 203.82 & 81.21 \\
\hline & \multirow{2}{*}{ Hembra } & L_total & 22.51 & 1.18 \\
\hline & & \begin{tabular}{|l|} 
Peso \\
\end{tabular} & 200.12 & 29.28 \\
\hline \multirow{4}{*}{$\begin{array}{l}\text { Basilio } \\
\text { Vadillo }\end{array}$} & \multirow[t]{2}{*}{ Macho } & L_total & 24.57 & 1.76 \\
\hline & & \begin{tabular}{|l|} 
Peso \\
\end{tabular} & 267.33 & 61.71 \\
\hline & \multirow{2}{*}{ Hembra } & L_total & 23.70 & 1.48 \\
\hline & & Peso & 250.41 & 45.20 \\
\hline \multirow{4}{*}{ El Salto } & \multirow{2}{*}{ Macho } & L_total & 26.71 & 2.46 \\
\hline & & \begin{tabular}{|l|} 
Peso \\
\end{tabular} & 375.53 & 100.61 \\
\hline & \multirow{2}{*}{ Hembra } & L_total & 26.56 & 2.14 \\
\hline & & Peso & 378.09 & 87.28 \\
\hline \multirow{4}{*}{ Calderón } & \multirow{2}{*}{ Macho } & L_total & 28.72 & 2.64 \\
\hline & & \begin{tabular}{|l|} 
Peso \\
\end{tabular} & \begin{tabular}{|l|}
486.78 \\
\end{tabular} & 123.33 \\
\hline & \multirow{2}{*}{ Hembra } & L_total & 30.71 & 4.30 \\
\hline & & Peso & 589.45 & 240.64 \\
\hline \multirow{4}{*}{ Cajititlán } & \multirow{2}{*}{ Macho } & L_total & 20.81 & 2.40 \\
\hline & & \begin{tabular}{|l|} 
Peso \\
\end{tabular} & 183.80 & 45.03 \\
\hline & \multirow{2}{*}{ Hembra } & L total & 21.05 & 1.96 \\
\hline & & Peso & 186.67 & 36.81 \\
\hline
\end{tabular}

Fuente: Elaboración propia

\section{Relación longitud-peso}

El análisis de las relaciones talla-peso fue mejor definido por el modelo potencial de la forma $\mathrm{W}=\mathrm{A} \mathrm{L}{ }^{\mathrm{b}}$. En la tabla 2 se muestran los valores de determinación $\left(\mathrm{R}^{2}\right)$ valor $\mathrm{F}$, grados de libertad, significación y los parámetros $a$ y $b$ de las ecuaciones para cada cuerpo de agua. 
Revista Iberoamericana de las Ciencias Biológicas y Agropecuarias

Tabla 2. Parámetros de las ecuaciones del modelo talla-peso por cuerpo de agua

\begin{tabular}{|c|c|c|c|c|c|c|}
\hline \multirow{2}{*}{ Sitios } & \multicolumn{4}{|c|}{ Resumen del modelo } & \multicolumn{2}{c|}{ Parámetros } \\
\cline { 2 - 7 } & $\mathrm{R}^{2}$ & $\mathrm{~F}$ & $\mathrm{gl}$ & $\mathrm{Sig}$ & $\mathrm{a}$ & $\mathrm{b}$ \\
\hline Zapotlán & 0.63 & 1770.21 & 1040 & 0.000 & 0.244 & 2.14 \\
\hline $\begin{array}{c}\text { Basilio } \\
\text { Vadillo }\end{array}$ & 0.644 & 1422.64 & 785 & 0.000 & 0.136 & 2.36 \\
\hline El Salto & 0.83 & 2514.91 & 514 & 0.000 & 0.042 & 2.78 \\
\hline Calderón & 0.875 & 4900.11 & 699 & 0.000 & 0.08 & 2.59 \\
\hline Cajititlán & 0.735 & 1610.51 & 580 & 0.000 & 0.314 & 2.1 \\
\hline
\end{tabular}

Fuente: Elaboración propia

Para todos los cuerpos de agua los coeficientes de determinación fueron bajos, pues varían de 0.875 para Calderón a 0.630 para Zapotlán. Los valores más bajos corresponden a Zapotlán, B. Vadillo y Cajititlán, con 0.63, 0.644 y 0.735, respectivamente (dos lagos naturales y una presa). Solo las presas El Salto y Calderón tuvieron determinaciones superiores a 0.8 , con 0.830 y 0.875 , respectivamente.

El parámetro $b$ es la base de la interpretación del modelo y representa la forma de crecimiento para cada población: isométrico $(2.5>\mathrm{b}<3.5)$, alométrico positivo $(\mathrm{b}>3)$, alométrico negativo ( $<3$ ). De acuerdo con los valores encontrados de $b$, todos los cuerpos de agua caen dentro del rango de alometria negativa $(b>3)$ y en solo dos casos se presentó isometría, es decir, valores mayores a 2.5, que corresponden nuevamente a las presas El Salto y Calderón (con 2.78 y 2.59, respectivamente), mientras que los valores más bajos fueron para los lagos Cajititlán, Zapotlán y la presa B. Vadillo, con 2.10, 2.13 y 2.36, respectivamente.

\section{Variables físico-químicas}

En los cinco cuerpos de agua la temperatura promedio fue de $22.75{ }^{\circ} \mathrm{C}$ e intervalos de 16.54 a 28.53. Los valores máximos se presentaron en julio-septiembre y los mínimos en noviembre-enero. Particularmente, con promedio de $25^{\circ} \mathrm{C}$, la presa Basilio Vadillo resultó ser la más cálida, con variaciones de más de 2 grados en casi todos los casos, seguida de Calderón, Zapotlán, Cajititlán y El Salto (los lagos naturales no presentaron diferencias significativas entre ellos). 
El sitio con mayor concentración de oxígeno disuelto en el agua fue Calderón. Igualmente, resaltan El Salto y B. Vadillo por sus amplias variaciones, mientras que los lagos se mantuvieron más o menos estables en relación con este factor y sin diferencias significativas. El análisis de correlación de Pearson entre las concentraciones de oxígeno y temperaturas, aunque bajas, fueron inversas en todos los casos: es decir, concentraciones altas de oxígeno se relacionan con bajas temperaturas. Los valores de correlación (r) para cada cuerpo de agua fueron -0.44, - -0.12, -0.04, -0.63, -0.78, en Zapotlán, B. Vadillo, El Salto, Calderón y Cajititlán, respectivamente, y solo en El Salto la relación no fue significativa.

Otro factor que resultó elevado (es decir, alcalino) en todos los sitios analizados fue el pH, pues las variaciones fueron amplias en las tres presas, mientras que en los lagos varió poco. Se encontraron diferencias significativas entre B. Vadillo y los lagos. Los sólidos totales disueltos presentaron promedio de $0.32 \mathrm{~g} / 1$, con mínimo de 0.13 y máximo de 0.69 $\mathrm{g} / \mathrm{l}$. Los promedios de los cuerpos de agua alcanzaron diferencias significativas $(\mathrm{p}<0.5)$ y los más altos para este factor fueron 0.63 y 0.4 g/l, que corresponden a los lagos Zapotlán y Cajititlán, respectivamente. Los sólidos en suspensión pueden aumentar la turbidez y disminuir también la concentración de oxígeno. Para el potencial de óxido-reducción solo en Calderón se presentaron valores positivos tanto individuales como en promedio (aunque bajos), mientras que en los demás cuerpos de agua los promedios estuvieron cercanos a cero en B. Vadillo y negativos en los sitios restantes (figura 2). 
Revista lberoamericana de las Ciencias Biológicas y Agropecuarias

Figura 2. Representación de los promedios \pm desviación de las variables ambientales

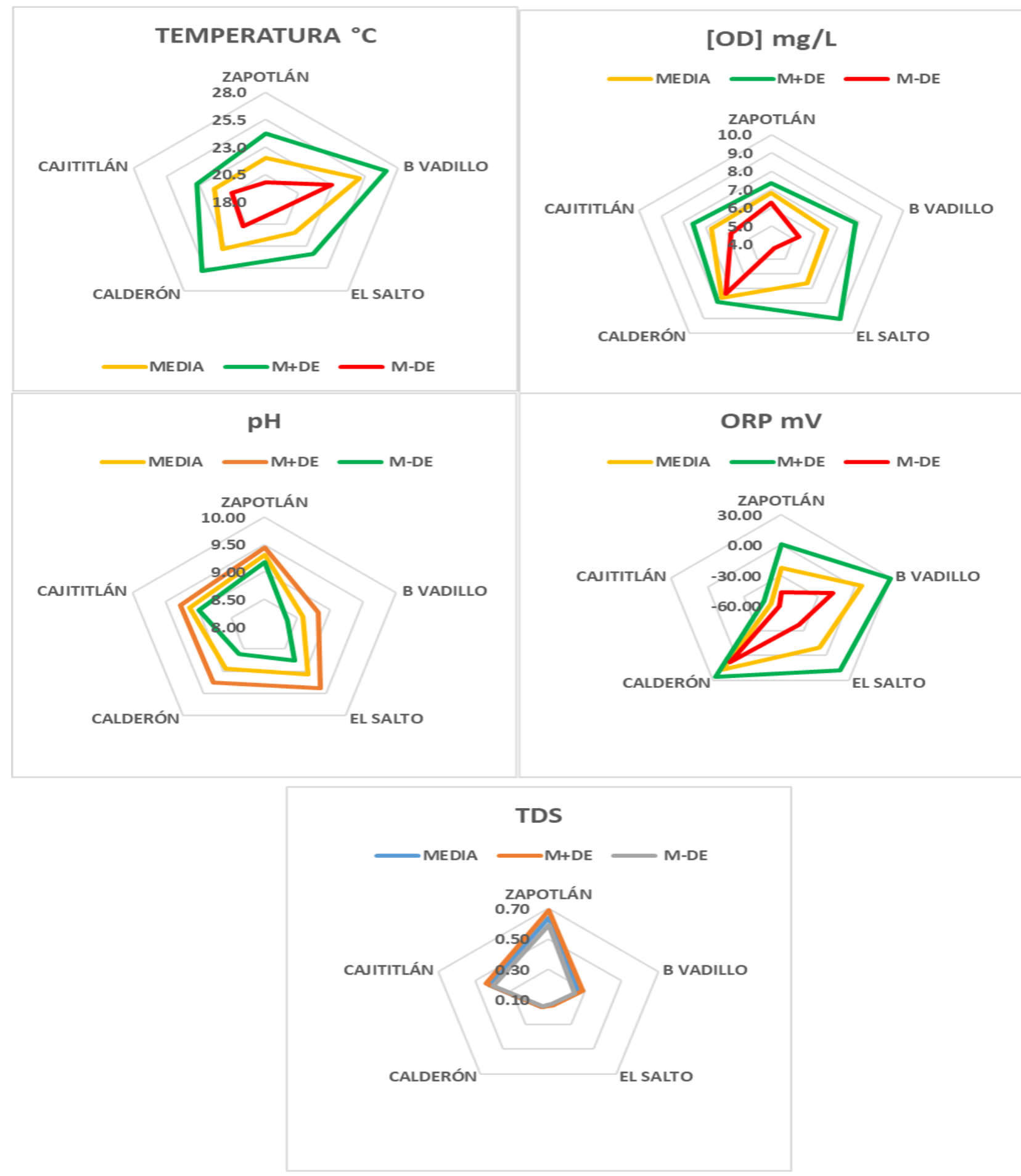

Fuente: Elaboración propia 


\section{Factor de condición de Fulton (K)}

Los valores promedio del factor de condición en todos los meses fueron bastante variables y con diferencias significativas en tiempo y por cuerpo de agua $(p<0,01)$ (tabla 3$)$.

Tabla 3. Prueba posthoc para factor de condición

\begin{tabular}{|l|l|l|l|l|l|l|}
\hline \multicolumn{2}{|c|}{ k_Fulton } \\
\hline & Lugar & \multicolumn{5}{|c|}{ Subconjunto para alfa $=.05$} \\
\cline { 2 - 7 } & & & & & \\
\hline \multirow{4}{*}{$\begin{array}{c}\text { HSD de } \\
\text { Tukey (a,b) }\end{array}$} & Zapotlán & 1.716 & & & & \\
\cline { 2 - 8 } & B. Vadillo & & 1.813 & & & \\
\cline { 2 - 8 } & El Salto & & & 1.959 & & \\
\cline { 2 - 7 } & Calderón & & & & 2.063 & \\
\cline { 2 - 7 } & Cajititlán & & & & & 2.101 \\
\hline
\end{tabular}

Fuente: Elaboración propia

Los promedios más bajos se presentaron en Zapotlán y B. Vadillo, mientras que los más altos en Calderón y Cajititlán. Las tendencias a descender temporalmente se presentaron en Calderón y B. Vadillo, a aumentar en El Salto y Cajititlán y a mantenerse siempre bajas en Zapotlán (figura 3).

Aunque las tallas de los organismos son bastante homogéneas, la variación individual del factor de condición exhibe un patrón aleatorio $\left(\mathrm{R}^{2}>0.33\right.$ en todos los casos), tendiente a disminuir con la talla en los cuerpos de agua (figura 3), pero con pendientes "suaves" en El Salto (-0.016) y Calderón (-0.045), respecto de pendientes más "fuertes" en B. Vadillo (0.056), Zapotlán (-0.072) y Cajititlán (-0.093), respectivamente (tabla 4). 
Revista Iberoamericana de las Ciencias Biológicas y Agropecuarias

Figura 3. Regresión lineal simple entre la talla y el factor de condición de Fulton $(\mathrm{K})$ en cada cuerpo de agua, en orden descendente de acuerdo con la pendiente (b)

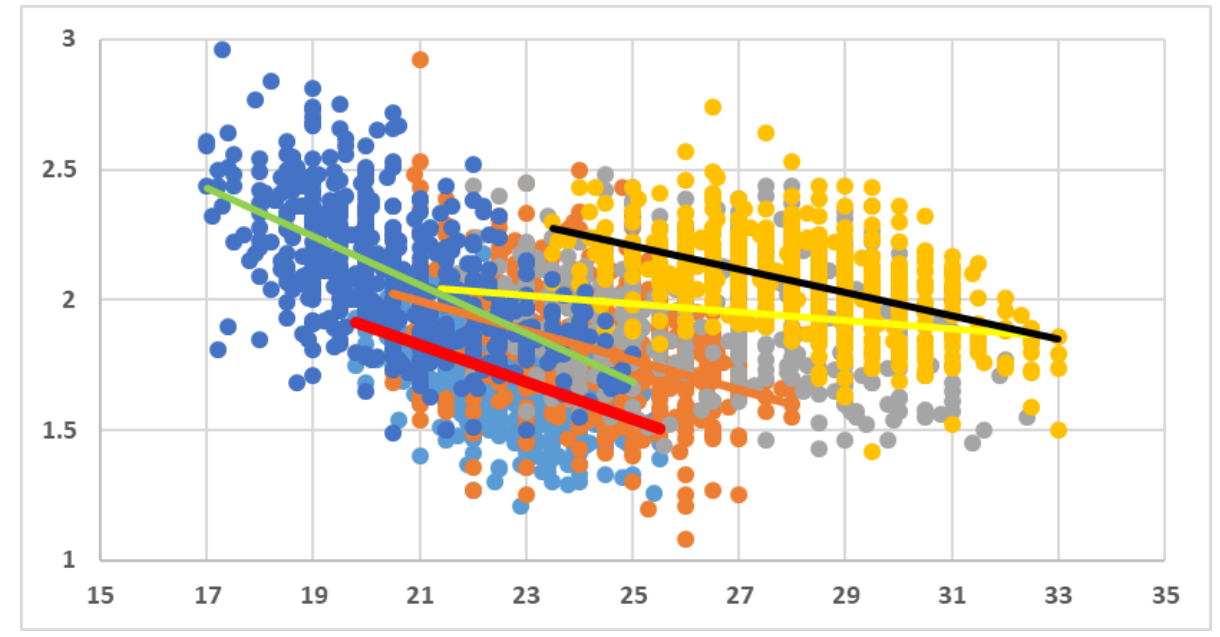

Fuente: Elaboración propia

Tabla 4. Parámetros del modelo factor de condición K y talla LT prueba poshoc para factor de condición tabla

\begin{tabular}{|c|c|c|}
\hline Sitio & Modelo lineal & $\mathrm{r}^{2}$ \\
\hline Cajititlán & $\mathrm{K}=-0.093 \mathrm{LT}+4.012$ & 0.331 \\
\hline Zapotlán & $\mathrm{K}=-0.072 \mathrm{LT}+3.337$ & 0.242 \\
\hline B. Vadillo & $\mathrm{K}=-0.056 \mathrm{LT}+3.180$ & 0.138 \\
\hline Calderón & $\mathrm{K}=-0.045 \mathrm{LT}+3.331$ & 0.235 \\
\hline El Salto: & $\mathrm{K}=-0.016 \mathrm{LT}+2.389$ & 0.032 \\
\hline
\end{tabular}

Fuente: Elaboración propia

La distribución temporal del factor de condición exhibe que los valores más bajos siempre se presentaron en Zapotlán; en B. Vadillo se registraron bajos en prelluvias y poslluvias e intermedios en lluvias; para El Salto también se presentaron valores intermedios en todas las muestras independientemente de la temporada; Calderón registró los valores más altos (independientemente de la temporada), mientras que Cajititlán tuvo valores altos en poslluvias e intermedios en las muestras restantes (figura 4). 
Revista Iberoamericana de las Ciencias Biológicas y Agropecuarias

Figura 4. Representación del factor de condición por lugar y temporada

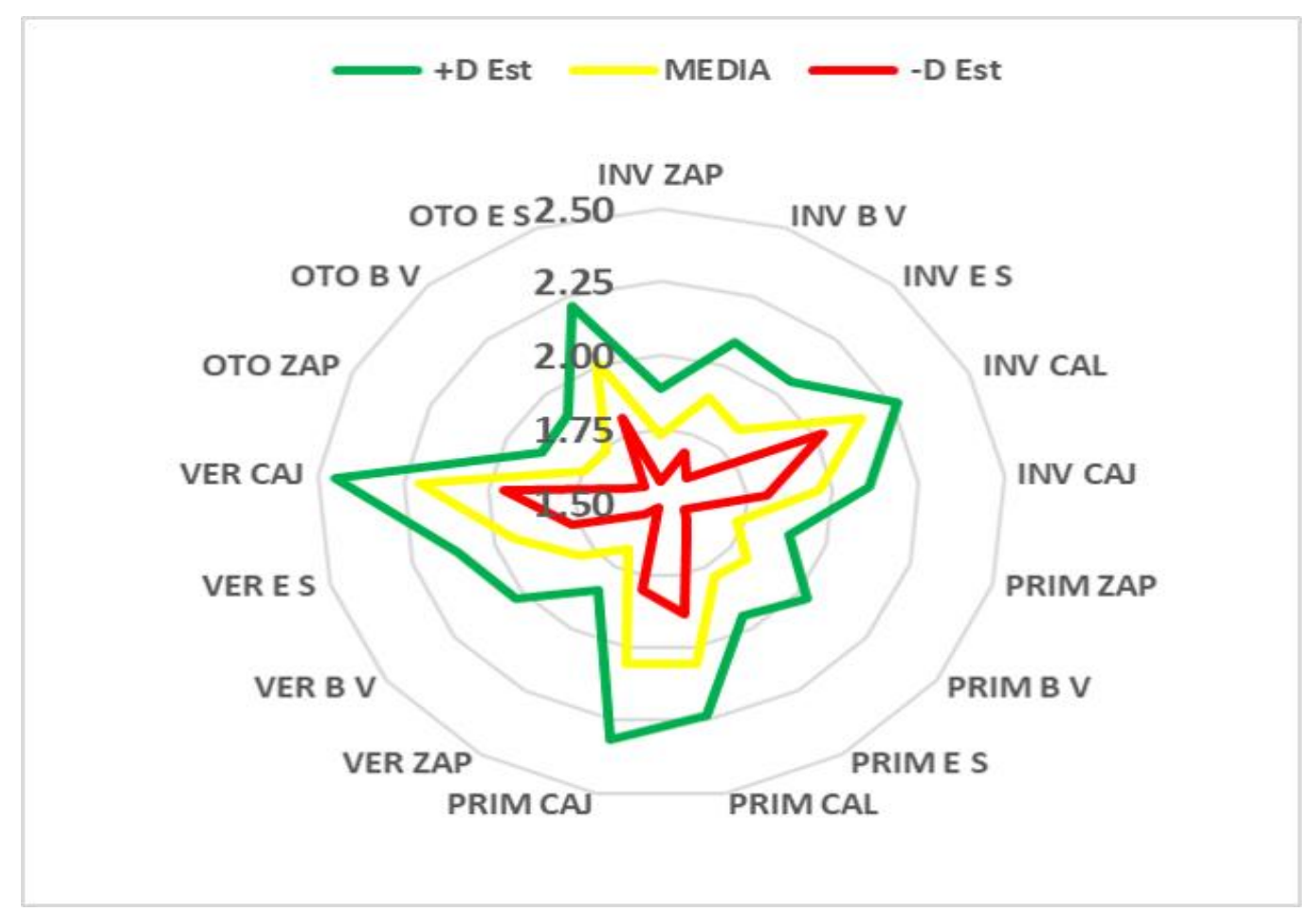

Fuente: Elaboración propia

\section{Relaciones entre factores ambientales y morfométricos}

El análisis de correlación entre variables ambientales y morfométricas exhibió relaciones significativas directas entre la talla y el peso $\left(\mathrm{r}^{2}=0.933\right)$, talla con ORP $(0.505)$, e inversas entre talla y TDS $\left(r^{2}=-0.656\right)$. El peso al estar altamente relacionado con la talla presentó también relaciones significativas con TDS y ORP (con $\mathrm{r}^{2}=-0.71$ y 0.496 , respectivamente). Por otra parte, aunque el factor de condición depende de las variables de talla y peso, solo exhibe relación inversa, muy tenue con TDS $r^{2}=-0.447$. En cuanto a los factores ambientales, el pH tuvo relación directa con TDS e inversa con ORP, mientras que entre TDS y ORP fue inversa (tabla 5). 
Revista Iberoamericana de las Ciencias Biológicas y Agropecuarias

Tabla 5. Análisis de correlación de Pearson entre variables morfométricas y ambientales

\begin{tabular}{|l|l|l|l|l|l|l|l|l|}
\hline & L_Total & Peso & K_Fulton & Temp & DO & pH & TDS & ORP \\
\hline L_Total & --- & $\mathbf{0 . 9 3 3}$ & -0.036 & 0.128 & 0.016 & -0.235 & $\mathbf{- 0 . 6 5 6}$ & $\mathbf{0 . 5 0 5}$ \\
\hline Peso & 0.0000 & --- & 0.299 & 0.082 & 0.04 & -0.208 & $\mathbf{- 0 . 7 1 0}$ & $\mathbf{0 . 4 9 6}$ \\
\hline K_Fulton & 0.037 & 0.0000 & --- & -0.099 & 0.101 & -0.091 & $\mathbf{- 0 . 4 4 7}$ & 0.134 \\
\hline Temp & 0.0000 & 0.0000 & 0.0000 & --- & -0.115 & -0.111 & -0.143 & -0.032 \\
\hline DO & 0.377 & 0.027 & 0.0000 & 0.0000 & --- & 0.234 & -0.112 & 0.095 \\
\hline pH & 0.0000 & 0.0000 & 0.0000 & 0.0000 & 0.0000 & --- & $\mathbf{0 . 4 9 6}$ & $\mathbf{- 0 . 4 8 2}$ \\
\hline TDS & 0.0000 & 0.0000 & 0.0000 & 0.0000 & 0.0000 & 0.0000 & --- & $\mathbf{- 0 . 4 9 1}$ \\
\hline ORP & 0.0000 & 0.0000 & 0.0000 & 0.076 & 0.0000 & 0.0000 & 0.0000 & --- \\
\hline
\end{tabular}

Fuente: Elaboración propia

\section{Discusión}

La tilapia es un pez exótico de origen tropical que tiene amplia tolerancia y adaptabilidad, con óptimos térmicos que van de $25-28{ }^{\circ} \mathrm{C}$ a $32{ }^{\circ} \mathrm{C}$ (Instituto Nacional de la Pesca [Inapesca], 2011). Por tanto, si disminuye a $20{ }^{\circ} \mathrm{C}$, dejan de comer; y si presentan cambios repentinos de $5{ }^{\circ} \mathrm{C}$ o descensos a $<12{ }^{\circ} \mathrm{C}$ pueden causar mortalidad (Inapesca, 2011).

Ahora bien, en todos los casos — con excepción de la presa B. Vadillo- se registraron mínimos $<20^{\circ} \mathrm{C}$, lo que indica — según los valores promedio de temperaturaque los cuerpos de agua analizados no alcanzan los valores óptimos para su desarrollo.

El oxígeno es fundamental en los sistemas acuáticos porque condiciona la distribución, el comportamiento y el crecimiento de los organismos (Wetzel, 2001). Oreochromis niloticus es tolerante también a bajas concentraciones de oxígeno > 3mg/l, aunque temperaturas superiores a $\operatorname{los} 30{ }^{\circ} \mathrm{C}$ aumenta su consumo, mientras que concentraciones menores a $5 \mathrm{mg} / \mathrm{l}$ y $6 \mathrm{mg} / \mathrm{l}$ hacen disminuir el metabolismo, el consumo de alimento y la saciedad, de modo que el crecimiento individual se hace más lento (Inapesca, 2011; Pandit y Nakamura, 2010). Por tanto, es probable que esto haya ocurrido al menos en las presas B. Vadillo y El Salto.

En el caso del Oreochromis que se halla bajo cultivo, se recomienda mantener concentraciones de oxígeno disuelto superiores a 5 ppm o 5 mg/l (Bautista y Ruiz-Velasco, 2011), ya que en condiciones experimentales, las concentraciones inferiores a $3 \mathrm{mg} / \mathrm{l}$ tienen como respuesta una menor ingesta de alimento; además, existe una relación directa entre el 
Revista Iberoamericana de las Ciencias Biológicas y Agropecuarias

tamaño corporal y la demanda o consumo (organismos más pequeños consumen más oxígeno). Asimismo, el consumo también se relaciona con conductas de alimentación: organismos a $3 \mathrm{mg} / \mathrm{l}$ consumen más alimento en la mañana, mientras que a $5.6 \mathrm{mg} / 1$ lo hacen más por la tarde (Tran, Ben, Dam y Schrama, 2008). Para el caso de los cuerpos de agua objeto de este estudio, solo El Salto mostró valores cercanos a $4 \mathrm{mg} / \mathrm{l}$, mientras que todos los demás fueron relativamente normales, es decir, por encima de $5 \mathrm{mg} / \mathrm{l}$.

La valoración de la calidad del hábitat acuático a partir de variables físico-químicas (que tengan un efecto directo en el comportamiento fisiológico de los organismos, como las utilizadas en este estudio) es una herramienta muy útil para demostrar los impactos antropogénicos en los cuerpos de agua. La medición de la temperatura que estableció niveles subóptimos de este factor para la especie, concentraciones estándar de oxígeno disuelto, $\mathrm{pH}$ bastante alcalino desviado del básico, altas cargas orgánicas que modifican los sólidos suspendidos totales y el potencial óxido reductor en los cuerpos de agua proveen información del grado de alteración en todos los casos estudiados del hábitat acuático (Orozco, Pérez, Gonzáles, Rodríguez y Alfayate, 2015); por otro lado, los índices bioecológicos aportan esta información, pero no señalan nada acerca de los factores responsables, por lo que es recomendable la utilización de ambos en la evaluación. Una de las ventajas de los métodos físico-químicos es su rapidez, por lo que pueden ser monitoreados con mayor frecuencia, a diferencia de los métodos biológicos, que exigen observaciones masivas y mediciones precisas de procesos bastante inciertos y de variación amplia que exhiben los seres vivos (Samboni, Escobar y Escobar, 2007), como los documentados en este estudio.

De acuerdo con los valores de temperatura encontrados en las presas El Salto y Calderón, así como en los lagos de Zapotlán y Cajititlán, permite clasificarlos como de aguas frías, mientras que las aguas de la presa B. Vadillo son un poco más cálidas. A diferencia de las tres presas, los lagos de Zapotlán y Cajititlán (de características endorreicas) presentan concentraciones de oxígeno parecidas a lagos eutróficos. Estas concentraciones se relacionan con valores muy bajos de OPR y altos de TDS y pH, como resultado de las altas cargas orgánicas, principalmente nitrógeno, amonio $\left(\mathrm{NH}_{3}\right)$ y fósforo, ya que son receptoras directas de aguas residuales industriales y urbanas de las poblaciones aledañas, además de otras fuentes difusas que resultan de la actividad agropecuaria. 
Revista Iberoamericana de las Ciencias Biológicas y Agropecuarias

Los factores de oxígeno disuelto, $\mathrm{pH}$, sólidos disueltos totales (TDS) y potencial óxido reducción (ORP) parecen estar relacionados con el número de habitantes en la cuenca y la distancia de las localidades urbanas a los sitios en que se tomaron las muestras: 18155 habitantes en la cuenca del río Calderón; 32220 habitantes en la cuenca donde se ubica la presa El Salto y más de 100000 habitantes en la cuenca donde se halla el lago de Zapotlán.

Las descargas tanto de nitrógeno como de fósforo constituyen un problema de gran magnitud para la supervivencia y sustentabilidad ecológica y económica de cuerpos de agua cercanos a zonas urbanas (Eugercios-Silva, Álvarez-Cobelas y Montero-González, 2017), ya que el nitrógeno en forma de amonio puede ser tóxico para los peces, incluso para las tilapias, a pesar de su resistencia y adaptabilidad. Por otra parte, nitratos y fosfatos intensifican el crecimiento de plantas y algas, lo que acelera la eutrofización de lagos y embalses (CamposPulido, Alonso-López, Avalos-de la Cruz, Asiain-Hoyos y Reta-Mendiola 2013), como lo muestran las coberturas del lirio Eichornia crassipes documentadas en el presente estudio (tabla 2).

Además, en bajas concentraciones de oxígeno disuelto los peces son más susceptibles a enfermedades, ya que la actividad metabólica se ve limitada (Tomalá, Chavarría y Escobar, 2014). En los lagos, los problemas se agravan al considerar la condición endorreica, ya que la única entrada de agua al sistema es de naturaleza pluvial (por lluvia), mientras que su única salida es por evaporación, lo que le confiere una dinámica de dilución y concentración temporal, aunque ligada al ciclo anual de precipitación (Bernal-Brooks, 2002). En cambio, en las presas este patrón de dilución-concentración se relaciona con su manejo, lo cual explica las diferencias entre los cinco cuerpos de agua de este estudio, así como las variaciones aleatorias individuales del factor de condición y las tendencias de esas variaciones en relación con la talla o peso, y/o lago o presa de que se trate.

Por último, se debe recalcar que en los lagos de Cajititlán y Zapotlán se enfrentan serios problemas de deterioro ambiental debido a la contaminación de residuos sólidos y el arrastre de agroquímicos (Secretaría del Medio Ambiente y Desarrollo Territorial [Semadet], 2015). Según estimaciones, en 2013 en Cajititlán se generaban $99.51 \mathrm{~s}^{-1}$ de aguas residuales, de las cuales se trataba $57.38 \%$ (es decir, $57.51 \mathrm{~s}^{-1}$ ), porcentaje que descendió a $50 \%$ en 2016 (CEA, 2016); mientras que en Zapotlán solo se trataba $75 \%$ de las aguas residuales de ciudad Guzmán (Santoyo, datos propios 2017), sin considerar otras poblaciones como 
Gómez Farías y San Andrés, que también vierten las aguas residuales a la laguna. Otros problemas que influyen en el deterioro de la cuenca y la calidad del hábitat acuático del lago Zapotlán son los producidos por las presiones derivadas de los cambios de uso de suelo — de forestal a urbano en la cuenca baja y de forestal a agropecuaria (huertas de aguacate y ganadería)_, así como la tala clandestina en la cuenca alta e inclusive dentro del parque nacional nevado de Colima (Semadet, 2015).

\section{Conclusiones}

Los cuerpos de agua que reciben descargas de aguas residuales urbanas sin el debido tratamiento mostraron tallas de peces más pequeñas, así como menor peso y longitud, especialmente en el caso de Cajititlán y Zapotlán, que se ubican en las inmediaciones de dos zonas urbanas (zona metropolitana de Guadalajara y zona metropolitana del Sur). Además, los cuerpos de agua de Cajititlán y Zapotlán comparten dos de las temperaturas promedio más bajas, los dos $\mathrm{pH}$ promedio más altos, las dos salinidades más elevadas, los dos TDS más significativos y los ORP más pequeños. En otras palabras, cuatro de las seis variables están en condiciones extremas en estos embalses.

En cuanto a las tilapias de mayor envergadura, estas se capturaron en las presas de El Salto y Calderón. Es importante mencionar que el factor de condición (K) de los peces varía dependiendo principalmente con el peso y la longitud, y en este estudio se relacionó estrechamente con las condiciones de contaminación de los cuerpos de agua.

Dado que las mediciones de oxígeno para este estudio fueron diurnas, se recomienda trabajar con otras en la parte oscura del día para establecer la dinámica del oxígeno y sus posibles implicaciones en el crecimiento de los peces, particularmente para los casos de Zapotlán y Cajititlán, los cuales mostraron altos niveles de distrofia. 


\section{Referencias}

Bagenal B. and Tesch, F. (1978). Age and growth. In Bagenal, T. B. (ed.). Methods for assessment of fish production in freshwaters (3 ${ }^{\text {th }}$ ed.) (pp. 101-136). Oxford: Blackwell Scientific publication.

Bautista, J. C. y Ruiz-Velazco, J. M. (2011). Calidad de agua para el cultivo de tilapia en tanques de geomembrana. Revista Fuente, 3(8), 10-14.

Bernal-Brooks, F. (2002). La limnología del Lago de Pátzcuaro: una visión alternativa a conceptos fundamentales (tesis de doctorado). México: Facultad de Ciencias. UNAM.

Campos-Pulido, R., Alonso-López, A., Avalos-de la Cruz, D. A., Asiain-Hoyos, A. y RetaMendiola, J. L. (213). Caracterización fisicoquímica de un efluente salobre de tilapia en acuaponia. Revista Mexicana de Ciencias Agrícolas, 4(5), 939-950.

Carlander, K. (1969). Handbook of freshwater fishery biology (vol. I). University Press, The Iowa State, EEUU.

Cifuentes, R., González, J., Montoya, G., Jara, A., Ortíz, N., Piedra, P. y Habit, E. (2012). Relación longitud-peso y factor de condición de los peces nativos del río San Pedro (cuenca del río Valdivia, Chile). Gayana (Concepción), 76(1), 86-100. Doi: https://dx.doi.org/10.4067/S0717-65382012000100009

Comisión Estatal del Agua del Estado de Jalisco [CEA] (2015). Ficha técnica hidrológica municipal.

Recuperado de: https://www.ceajalisco.gob.mx/doc/fichas_hidrologicas/region9/zapotlan\%20el\%20 grande.pdf.

Cotler, H. (coord.) (2010). Las cuencas hidrográficas de México diagnóstico y priorización. México: Pluralia Ediciones. Recuperado de https://agua.org.mx/wpcontent/uploads/2011/02/CuencasHidrogra\%CC\%81ficas-1.pdf.

Da-Costa, M. and Gerson, F. (2003). Length-weight relationship and condition factor of Micropogonias furnieri (Desmarest) (Perciformes, Sciaenidae) in the Sepetibia Bay, Rio de Janeiro State, Brazil. Revista Brasileria de Zoología, 20(4), 685-690.

Eugercios-Silva, A. R., Álvarez-Cobelas, M. y Montero González, E. (2017). Impactos del nitrógeno agrícola en los ecosistemas acuáticos. Ecosistemas, 26(1), 37-44.

Froese, R. (2006). Cube law, condition factor and weight-length relationships: history, metaanalysis and recommendations. Journal of Applied Ichthyology, 22, 241-253. 
Revista Iberoamericana de las Ciencias Biológicas y Agropecuarias

Instituto Nacional de Estadística, Geografía e Informática (Inegi) (2000). Estudio hidrológico del estado de Jalisco. Recuperado de http://internet.contenidos.inegi.org.mx/contenidos/productos/prod_serv/contenidos/e spanol/bvinegi/productos/historicos/2104/702825221751/702825221751_1.pdf\#[38, $\{\% 22$ name\%22:\%22Fit\%22\}

Instituto Nacional de la Pesca [Inapesca] (2011). Acuacultura comercial. Recuperado de https://www.gob.mx/inapesca/acciones-y-programas/acuacultura-tilapia.

Martínez, L. (1987). Métodos de evaluación, control y racionamiento en la alimentación práctica. Alimentación en Acuicultura. Comisión Asesora de Investigación Científica y Técnica. Madrid: Espinoza y Labarta Editores.

Orozco C., Pérez A., Gonzáles M., Rodríguez F. y Alfayate, J. (2015). Contaminación ambiental. Una visión desde la química (3. ${ }^{\mathrm{a}}$ ed.). España: Editorial Paraninfo.

Pandit, N. and Nakamura, M. (2010). Effect of High Temperature on Survival, Growth and Feed Conversion Ratio of Nile Tilapia, Oreochromis niloticus. Our Nature, 8(1), 219224. https://doi.org/10.3126/on.v8i1.4331

Ricker, W. (1975). Computation and interpretation of biological statistics of fish populations. Bulletin of the Fisheries Research Board of Canada, 191, 1- 382.

Samboni, N., Escobar, Y. y Escobar, J. (2007). Revisión de parámetros físicoquímicos como indicadores de calidad y contaminación del agua. Revista Ingeniería e Investigación, 27(3), 172-181.

Secretaría del Medio Ambiente y desarrollo territorial [Semadet] (2010). Atiende SEMADET problemáticas ambientales de Laguna de Zapotlán El Grande. http://www.jalisco.gob.mx/es/prensa/noticias/21011.

Tomalá, J., Chavarría, L. y Escobar. B (2014). Evaluación de la tasa de consumo de oxígeno de Colossoma macropomum en relación al peso corporal y temperatura del agua. Latin American Journal of Aquatic Research, 42(5), 971-979. Doi: 10.3856/vol42issue 5-fulltext-4

Tran, D., Ben, S., Dam, A. and Schrama, W. (2008). Effects of dietary starch and energy levels on maximum feed intake, growth and metabolism of Nile tilapia, Oreochromis niloticus. $\quad$ Aquaculture, 277(3-4), 213-219. Doi https://doi.org/10.1016/j.aquaculture.2008.03.004 
Valencia-Santana, F. y Valencia-Santana, J. (2015). Relación longitud-peso y factor de condición de Echinometravanbrunti (A. Agassiz 1863) en Acapulco, Guerrero, México. Revista de Análisis Cuantitativo y Estadístico, 2(5), 402-407.

Wetzel, R. G. (2001). Limnología, ecología de lagos y ríos (3. a ed.). EUA: Ed. Academic Press.

\begin{tabular}{|l|l|}
\hline Rol de Contribución & Autor (es) \\
\hline Conceptualización & Felipe Santoyo Telles (Igual) y Carlos Gómez Galindo (Igual) \\
\hline Metodología & Carlos Gómez Galindo (Igual) y José Mariscal Romero (Igual) \\
\hline Software & Humberto Gutiérrez Pulido \\
\hline Validación & $\begin{array}{l}\text { Humberto Gutiérrez Pulido (Igual) y José Mariscal Romero } \\
\text { (Igual). }\end{array}$ \\
\hline Análisis Formal & Humberto Gutiérrez Pulido. \\
\hline Investigación & Felipe Santoyo Telles (Igual) y Carlos Gómez Galindo (Igual) \\
\hline Curación de datos & Humberto Gutiérrez Pulido (Igual) y Felipe Santoyo (Igual). \\
\hline $\begin{array}{l}\text { Escritura - Preparación del } \\
\text { borrador original }\end{array}$ & Felipe Santoyo Telles (Igual) y Carlos Gómez Galindo (Igual) \\
\hline $\begin{array}{l}\text { Escritura - Revisión y } \\
\text { edición }\end{array}$ & $\begin{array}{l}\text { José Mariscal Romero (Igual) y Humberto Gutiérrez Pulido } \\
\text { (Igual). }\end{array}$ \\
\hline Visualización & Felipe Santoyo Telles. \\
\hline Adquisición de fondos & Felipe Santoyo Telles. \\
\hline
\end{tabular}

